ISSN 2525-4804

\title{
PRODUÇÃO DE MUDAS DE BATATA-DOCE EM CLIMA TROPICAL
}

\section{Vanessa Ferreira Vilete ${ }^{1}$, Willian Pereira do Nascimento ${ }^{2}$, Thayla Rocha Aguirre ${ }^{3}$, Celso Pereira de} Oliveira $^{4}$

\section{RESUMO:}

A batata-doce (Ipomoea batatas) é uma hortaliça dicotiledônea que possui diversas variedades, além de fácil adaptação em clima tropical. O desenvolvimento inicial das mudas pode variar de acordo com a cultivar e sofrer alteração no seu desenvolvimento em razão das condições climáticas. Objetivou-se neste trabalho avaliar mudas de cinco cultivares de batata doce cultivadas no município de Ji-Paraná, Rondônia. O delineamento experimental foi inteiramente casualizado, composto por cinco tratamentos, sendo estes as cultivares JNRX-1, JNRX-12, JN-branca 1 e Canadense, adquiridas no Estado de São Paulo, e da cultivar Beauregard, adquirida na região local. Para a produção de mudas, a rama de plantas matrizes foi cortada e o material seccionado de forma que apresentasse uma folha acompanhada de uma gema e um fragmento de caule. Este material foi colocado para enraizar em frascos transparentes contendo água potável em temperatura ambiente. Três dias após, o material foi retirado do recipiente com água e repassado para sacos plásticos contendo substrato comercial para hortaliças, e identificadas de acordo com cada cultivar. Aos 30 dias após o preparo das mudas foi analisado: comprimento da raiz $(\mathrm{cm})$, comprimento da parte aérea $(\mathrm{cm})$, massa fresca da raiz $(\mathrm{g})$, massa fresca da parte aérea $(\mathrm{g})$, número de folhas, número de ramos e área foliar $\left(\mathrm{cm}^{2}\right)$. As cultivares adquiridas no estado de São Paulo (JNRX- 12, JNRX-1, JN Branca 1 e Canadense) se desenvolveram bem nas condições climáticas do município de Ji-Paraná/O, expressando valores semelhantes ou até mesmo superiores a cultivar Beauregard adquirida na região local. De acordo com as características avaliadas, nas condições climáticas do município, recomenda-se para a produção de mudas as cultivares JNRX-12, JN Branca 1 e Canadense, pois estas cultivares apresentaram maior comprimento e massa fresca de raiz e maior comprimento da parte aérea.

Palavras-chave: condições climáticas, Ipomoea batatas, propagação.

\section{SWEET POTATO SEEDLINGS PRODUCTION IN TROPICAL CLIMATE}

\begin{abstract}
:
The sweet potato (Ipomoea potatoes) is a dicotyledonous vegetable with several varieties that adapt well to tropical climates. The initial development of seedlings can vary according to the cultivar and the climatic conditions during seedling development. The objective of this study was to evaluate seedlings of five

\footnotetext{
${ }^{1}$ Graduanda em Agronomia, Centro Universitário São Lucas, Ji - Paraná - Rondônia; viletevanessa53@ gmail.com

${ }^{2}$ Graduando em Agronomia, Centro Universitário São Lucas, Ji - Paraná - Rondônia; eng.willian.nascimento@gmail.com

${ }^{3}$ Graduanda em Agronomia, Centro Universitário São Lucas, Ji - Paraná - Rondônia; aguirrethayla@gmail.com

${ }^{4}$ Eng. Agrônomo, Mestre em Olericultura; Pesquisador e Docente do Centro Universitário São Lucas, Ji - Paraná - Rondônia; celso.oliveira@saolucas.edu.br
} 
sweet potato cultivars grown in the municipality of Ji-Paraná, Rondônia. The experimental design was completely randomized. It was composed of five treatments: the cultivars JNRX-1, JNRX-12, JN-White 1, and Canadian, acquired in the State of São Paulo, and the cultivar Beauregard, acquired in the local region. For the seedlings production, the branch plant matrix was cut, and the material was sectioned so that it presented a leaf accompanied by yolk and a fragment of the stem. This material was placed in transparent flasks containing drinking water at room temperature to root. Three days later, the material was removed from the container with water; it was then transferred to plastic bags containing a commercial substrate for vegetables and identified according to each cultivar. At 30 days after seedling preparation, the study analyzed: root length $(\mathrm{cm})$, shoot length $(\mathrm{cm})$, fresh root weight $(\mathrm{g})$, fresh shoot weight $(\mathrm{g})$, number of leaves, number of branches, and leaf area $\left(\mathrm{cm}^{2}\right)$. The cultivars acquired in the state of São Paulo (JNRX-12, JNRX-1, JN White 1, and Canadian) developed well in the municipality's climatic conditions Ji-Paraná / Rondônia. They expressed similar or even higher values than cultivar Beauregard acquired in the local region. Results showed that the cultivars JNRX-12, JN White 1, and Canadian are recommended for seedlings production. This recommendation is due to the climatic conditions of the municipality of Ji-Paraná/RO. According to the evaluated characteristics, these cultivars presented longer root length, greater fresh mass root, and longer shoot length.

KeyWords: climatic conditions, Ipomoea potatoes, propagation. 


\section{INTRODUÇÃO}

A batata-doce (Ipomoea batatas) é classificada como uma hortaliça dicotiledônea da família das Convolvulaceae (Silva et al., 2008). Tem função alimentícia tanto para humanos, quanto para animais e recentemente tem seu uso destinado à produção de etanol (Camargo et al., 2016). Possui diversas variedades, sendo classificada conforme as seguintes características: coloração da polpa e da casca, tamanho, formato, e cor de flores e folhas (Mirasse, 2010; Furlaneto et al., 2012).

A cultura da batata-doce é implantada a partir de mudas feitas com material vegetativo, comumente utilizando-se partes da rama, sendo estas: velhas, quando retirada de culturas já implantadas, e novas, quando retiradas de viveiros (Filgueira, 2008).

No Brasil, o plantio e venda da batata-doce é uma atividade lucrativa (Melo et al., 2009). Segundo dados do Instituto Brasileiro de Geografia e Estatística- IBGE (2019), o Brasil no ano de 2018 chegou a produzir 741.203 toneladas de batata-doce com uma área plantada de 53.024 hectares, sendo o Rio Grande do Sul o maior estado produtor de batatadoce em território nacional, com uma produção de 175.060 toneladas neste mesmo ano, representando aproximadamente $24 \%$ do total.

O cultivo de batata-doce no Brasil é em grande parte realizado em um sistema de agricultura familiar, em que a produção caracteriza-se pelo baixo nível de tecnologia, desde a fase de propagação, onde o plantio se dá com o plantio de ramas (estacas), sem prévio enraizamento, diretamente a campo. Esse método de plantio direto das estacas a campo tem como desvantagem o alto índice de mortalidade, já que a secção do caule possui poucas reservas de nutriente aliada com condições ambientais desfavoráveis ao enraizamento o que, por conseguinte, gera a necessidade de replantio das mudas (Silva et al., 1995).

Sendo assim, o plantio de mudas préenraizadas por estaquia pode ser uma alternativa eficaz para a obtenção de mudas com uma qualidade superior ou quando não se tem muito material propagativo (Rós e Narita, 2011).

Para que seja feita a produção de mudas, é necessário o uso de recipientes como tubetes, bandejas, saquinho plástico para mudas ou até mesmo copos descartáveis que permitem um maior cuidado com as plântulas. Segundo Reghin et al. (2007), esse método facilita tanto o manejo nutricional quanto o cuidado com pragas e doenças, e por consequência aumenta a taxa de sobrevivência e maior uniformidade no campo.

Os recipientes têm como objetivos principais a proteção das raízes por eventuais danos mecânicos, alocação do substrato, maior taxa de sobrevivência e de desenvolvimento inicial da muda, além de evitar sua desidratação (Lisboa et al., 2012).

Esta cultura adapta-se bem em regiões de clima tropical (Neumann et al., 2017), em locais em que temperaturas médias são acima de $24{ }^{\circ} \mathrm{C}$, com alta luminosidade e com suficiente umidade do solo são capazes de expressar seu potencial vegetativo, e posteriormente produtivo (Silva et al., 2008).

O desenvolvimento inicial de batata-doce pode variar entre as cultivares, além da possibilidade de sofrer alteração em seu desenvolvimento por conta das condições climáticas (Rós-Golla et al., 2008).

Sendo assim, objetivou-se neste trabalho avaliar o desenvolvimento de mudas de cultivares de batata-doce adquiridas no Estado de São Paulo (JNRX-1, JNRX-12, JN-branca 1 e Canadense) e de uma cultivar adquirida na região local (Beauregard), cultivadas sobre condições climáticas do município de Ji- Paraná/Rondônia.

\section{MATERIAL E MÉTODOS}

O experimento foi conduzido no mês de Janeiro de 2020 na área experimental do Centro Universitário São Lucas de Ji-Paraná, na cidade de JiParaná/Rondônia, que está localizada sob as coordenadas geográficas de latitude: $10^{\circ} 57^{\prime} 28^{\prime \prime}$ sul e longitude $61^{\circ} 54^{\prime} 21^{\prime \prime}$ oeste, com altitude 156 metros na Amazônia Ocidental. O clima predominante em Rondônia é o tropical do tipo Aw- Tropical Chuvoso 
da classificação de KOPPEN, com temperaturas médias variando de $18^{\circ} \mathrm{C}$ a $26^{\circ} \mathrm{C}$, e estação seca bem acentuada, com precipitação pluviométrica variando de 1200 a 2600 mm/ano (Sedam, 2014).

O delineamento experimental foi inteiramente casualizado, com sete repetições, sendo os tratamentos realizados com cinco cultivares de batata-doce: JNRX-1, JNRX-12, JN-branca 1, Canadense e Beauregard.

A produção de mudas foi feita conforme Castro e Becker (2011). A rama de plantas matrizes foi cortada à altura de quatro a seis folhas, a partir da base, e o material seccionado de forma que apresentasse uma folha acompanhada de uma gema e um fragmento de caule com aproximadamente $1 \mathrm{~cm}$ de comprimento.

Em seguida, este material foi colocado para enraizar em frascos transparentes contendo cerca 200 ml de água potável em temperatura ambiente, tomando-se o cuidado para que apenas o caule tivesse o contato com a água, evitando-se as folhas, para diminuir o índice de apodrecimento das mudas. Três dias após, o material foi retirado do recipiente com água e repassado para sacos plásticos contendo $460 \mathrm{~g}$ de substrato comercial para hortaliças, sendo adição de adubos. Cada saco plástico foi identificado de acordo com cada cultivar.

As mudas permaneceram a campo, e irrigações diárias no período da tarde foram realizadas manualmente com auxílio de regador. Sempre que necessário foram realizadas a retirada manual de plantas invasoras. Não foram detectados ataques de pragas e doenças. Durante o período de condução do experimento foram registradas temperaturas mínimas de $23^{\circ} \mathrm{C}$ e máxima de $32^{\circ} \mathrm{C}$, com precipitação diária mínima de 2,0 mm e máxima de 20,1 mm (Accuweather, 2020).

Aos 30 dias após o preparo das mudas foi avaliado: a) comprimento de raiz $(\mathrm{cm})$ (Santana et al., 2015); b) comprimento da parte aérea $(\mathrm{cm})$ (Neumann et al., 2017); c) massa fresca de raiz (g) (Neumann et al., 2017); d) massa fresca da parte aérea (g)
(Neumann et al., 2017); e) número de folhas (Rós et al., 2013); f) número de ramos e g) área foliar $\left(\mathrm{cm}^{2}\right)$ (Ferreira et al., 2017).

Para as avaliações, as mudas foram levadas a laboratório onde foram retiradas dos sacos plásticos e retirado o excesso de substrato. Para medir o comprimento da raiz foi utilizada régua graduada, tendo como base para aferição do colo da planta ao ápice radicular (Santana et al., 2015). O comprimento da parte aérea foi medido utilizando régua graduada, mensurando-se desde o colo até o ápice da planta (Neumann et al., 2017). A massa fresca de raiz foi determinada em balança analítica, após lavagem da planta em água corrente e com posterior secagem em temperatura ambiente para evaporação da umidade adquirida pela lavagem (Neumann et al., 2017). A massa fresca da parte aérea foi determinada pesando em balança analítica toda parte aérea da muda (caule e folhas) (Neumann et al., 2017). Em seguida foi realizado contagem do número de folhas, considerando aquelas totalmente abertas, e número de ramos, considerando as brotações emitidas após o preparo das mudas (Rós et al., 2013). Para a determinação da área foliar foi utilizado o software DDA versão 1.2 (Determinador Digital de Áreas), onde foi escolhido a maior folha de cada muda para se realizar o escaneamento (Ferreira et al., 2017).

Os dados obtidos foram submetidos à análise de variância (ANAVA) para cada característica avaliada, sendo as médias comparadas pelo teste de Scott-Knott ao nível de $5 \%$ de probabilidade, utilizando o software Sisvar versão 5.6 (Ferreira, 2011).

\section{RESULTADOS E DISCUSSÃO}

$\mathrm{Na}$ Tabela 1, pode-se observar que foram verificadas diferenças significativas entre as cultivares apenas para as características comprimento de raiz, comprimento da parte aérea e massa fresca da raiz. 
Tabela 1. Resumo da análise de variância referente aos tratamentos de 30 dias após o preparo de mudas, do comprimento de raiz (C.R), comprimento da parte aérea (C.A), massa fresca de raiz (M.F.R), massa fresca da parte aérea (M.F.A), número de folhas (N.F), área foliar (A.F) e número de ramos (N.R) de mudas de cultivares de batata doce cultivadas no município de Ji-Paraná, Rondônia, 2020.

\begin{tabular}{lcccccccc}
\hline \multirow{2}{*}{$\begin{array}{l}\text { FONTES DE } \\
\text { VARIAÇÃO }\end{array}$} & GL & $\begin{array}{c}\text { C.R } \\
(\mathbf{c m})\end{array}$ & $\begin{array}{c}\text { C.A } \\
(\mathbf{c m})\end{array}$ & $\begin{array}{c}\text { M.F.R } \\
(\mathbf{g})\end{array}$ & $\begin{array}{c}\text { M.F.A } \\
(\mathbf{g})\end{array}$ & $\mathbf{N . F}$ & $\begin{array}{c}\text { A.F } \\
\left(\mathbf{c m}^{2}\right)\end{array}$ & NR \\
\hline TRATAMENTOS & 4 & $406,21^{*}$ & $114,45^{*}$ & $1,75^{*}$ & $5,17^{*}$ & $5,04^{\text {ns }}$ & $16,38^{\text {ns }}$ & $0,00^{\text {ns }}$ \\
BLOCOS & 6 & $131,92^{\text {ns }}$ & $9,53^{\text {ns }}$ & $0,91^{\text {ns }}$ & $1,11^{\text {ns }}$ & $4,84^{\text {ns }}$ & $36,11^{\text {ns }}$ & $0,00^{\text {ns }}$ \\
RESIDUOS & 24 & 63,94 & 40.22 & 0,64 & 2,30 & 4,27 & 21,85 & 0,00 \\
\hline Média & - & 40,71 & 13,60 & 2,34 & 3,54 & 6,65 & 12,74 & 1,00 \\
\hline C.V. $(\%)$ & - & 19,64 & 46,63 & 34,16 & 42,85 & 31,06 & 36,68 & 00,00 \\
\hline
\end{tabular}

* significativo ao nível de $5 \%$ de probabilidade $(\mathrm{p}<0.05)$ ns não significativo ( $\mathrm{p}>=0.05$ ).

Segundo dados da tabela 2, o maior comprimento de raiz foi expresso nas cultivares JNRX-12 (49,57 cm), JN-branca $1(43,00 \mathrm{~cm})$ e Canadense $(45,42 \mathrm{~cm})$. As cultivares JNRX-1 e Beauregard apresentaram respectivamente, $33,28 \mathrm{~cm}$ e 32,28 cm de comprimento de raíz.

As cultivares JN-branca 1 e JNRX-12 produziram maior massa fresca de raiz (como mostra a Tabela 2), onde ambas apresentaram 2,85 g. As cultivares JNRX-1 e Canadense obtiveram 2,14 g de massa fresca de raiz, enquanto a Beauregard 1,71 g.

Neunfeld (2019) avaliando mudas de trinta e sete acessos diferentes de batata doce, obteve valores entre $5,94 \mathrm{~cm}$ e $26,10 \mathrm{~cm}$ de comprimento de raiz e $0,04 \mathrm{~g}$ a $1,13 \mathrm{~g}$ de massa fresca da raiz, e embora tenha sido acessos diferentes e realizado a avaliação ao 75 dias após o preparo das mudas, apresentou resultados inferiores ao do presente estudo que foi avaliado aos 30 dias.

Tabela 2. Avaliação, 30 dias após o preparo de mudas, do comprimento de raiz (C.R), comprimento da parte aérea (C.A), massa fresca de raiz (M.F.R), massa fresca da parte aérea (M.F.A), número de folhas (N.F), área foliar (A.F) e número de ramos (N.R) de mudas de cultivares de batata doce cultivadas no município de JiParaná, Rondônia, 2020.

\begin{tabular}{cccccccc}
\hline Cultivares & $\begin{array}{c}\text { C.R } \\
(\mathbf{c m})\end{array}$ & $\begin{array}{c}\text { C.A } \\
(\mathbf{c m})\end{array}$ & $\begin{array}{c}\text { M.F.R } \\
(\mathbf{g})\end{array}$ & $\begin{array}{c}\text { M.F.A } \\
(\mathbf{g})\end{array}$ & $\mathbf{N . F}$ & $\begin{array}{c}\text { A.F } \\
\left(\mathbf{c m}^{2}\right)\end{array}$ & NR \\
\hline JNRX- 1 & $33,28 \mathrm{~b}$ & $10,42 \mathrm{~b}$ & $02,14 \mathrm{~b}$ & $02,42 \mathrm{a}$ & $05,57 \mathrm{a}$ & $13,42 \mathrm{a}$ & $01,00 \mathrm{a}$ \\
JNRX-12 & $49,57 \mathrm{a}$ & $19,00 \mathrm{a}$ & $02,85 \mathrm{a}$ & $04,42 \mathrm{a}$ & $07,00 \mathrm{a}$ & $12,85 \mathrm{a}$ & $01,00 \mathrm{a}$ \\
JN-branca 1 & $43,00 \mathrm{a}$ & $16,28 \mathrm{a}$ & $02,85 \mathrm{a}$ & $04,14 \mathrm{a}$ & $06,57 \mathrm{a}$ & $14,14 \mathrm{a}$ & $01,00 \mathrm{a}$ \\
Canadense & $45,42 \mathrm{a}$ & $13,00 \mathrm{~b}$ & $02,14 \mathrm{~b}$ & $03,85 \mathrm{a}$ & $07,85 \mathrm{a}$ & $10,14 \mathrm{a}$ & $01,00 \mathrm{a}$ \\
Beauregard & $32,28 \mathrm{~b}$ & $9,28 \mathrm{~b}$ & $01,71 \mathrm{~b}$ & $02,85 \mathrm{a}$ & $06,28 \mathrm{a}$ & $13,14 \mathrm{a}$ & $01,00 \mathrm{a}$ \\
\hline CV (\%) & 19,64 & 46,63 & 34,16 & 42,85 & 31,06 & 36,68 & 00,00 \\
F & 06,35 & 02,84 & 02,74 & 02,24 & 01,17 & 0,750 & 00,00 \\
\hline
\end{tabular}

Médias seguidas da mesma letra não diferem significativamente entre si pelo teste de Scott- Knott ao nível de 5\% de probabilidade; $\mathrm{CV}=$ coeficiente de variação; $\mathrm{F}=$ teste $\mathrm{F}$ da ANAVA. 
Segundo Rós-Golla et al. (2008), diferenças entre cultivares podem estar relacionadas às características genéticas, como por exemplo, formato das raízes, explicando assim o fato de algumas cultivares expressarem maior comprimento e massa fresca das raízes do que as demais cultivares.

Em relação ao comprimento da parte aérea (Tabela 2), os maiores valores obtidos foram das cultivares JNRX-12 (19,00 cm) e JN-branca $1(16,28$ $\mathrm{cm})$, enquanto as demais apresentaram resultados inferiores, JNRX-1 (10,42 cm), Canadense $(13,00$ $\mathrm{cm})$ e Beauregard $(9,28 \mathrm{~cm})$. Os valores obtidos estão de acordo com o padrão para comercialização de mudas, pois, segundo Castro e Becker (2011), as mudas que forem comercializadas devem possuir tamanho em média entre $10 \mathrm{~cm}$ e $20 \mathrm{~cm}$.

Estes valores são superiores ao encontrados por Neunfeld (2019), que, em clima temperado, aos 30 dias obteve valores de comprimento da parte aérea variando de $2,23 \mathrm{~cm}$ a $6,94 \mathrm{~cm}$. Vendruscolo et al. (2017) em Goiânia/GO, verificando o efeito de solução nutritiva e substrato turfoso em mudas de batata doce aos 28 dias após a estaquia, obtiveram altura de $1,85 \mathrm{~cm}$ a $4,93 \mathrm{~cm}$. O fato do presente estudo ter obtido dados superiores pode estar relacionadas às condições climáticas, onde a temperatura pode ter influenciado o desenvolvimento das plantas, pois durante o experimento a temperatura média foi de $27^{\circ} \mathrm{C}$ (AccuWeather, 2020). Estando assim, de acordo com Castro e Becker (2011) que recomendam temperaturas entre $22^{\circ} \mathrm{C}$ e $28^{\circ} \mathrm{C}$, para haver um bom desenvolvimento das mudas.

As cinco cultivares apresentaram apenas um ramo, número de folhas variando de 5,57 a 7,85, e área foliar entre $10,14 \mathrm{~cm}^{2}$ e $14,14 \mathrm{~cm}^{2}$. Vendruscolo et al. (2017), na produção de mudas com diferentes substratos, o número de folhas verdadeiras alcançou 2,61 folhas em média com o substrato turfoso em relação a outros tratamentos.

De acordo com Silva et al. (2008), uma característica que deve ser levada em consideração no momento de escolha de uma cultivar é o tamanho das folhas Isso é importante porque cultivares com folhas pequenas e estreitas são mais recomendadas, pois estas são mais eficientes na assimilação de energia na fotossíntese. Contrário a isso, as folhas grandes superiores geralmente sombreiam as inferiores interferindo no processo de assimilação.

\section{CONCLUSÃO}

De acordo com as características avaliadas, nas condições climáticas do município de JiParaná/RO, é recomendado para a produção de mudas as cultivares JNRX-12, JN-branca 1 e Canadense, pois estas cultivares apresentaram maior comprimento de raiz, maior massa fresca de raiz e maior comprimento da parte aérea.

\section{REFFERÊNCIAS}

AccuWeather. (2020). Condições meteorológicas de ji- paraná. ACCUWEATHER. Disponível em: https://www.accuweather.com/pt/br/jiparan\%C3\%A1/35942/january-weather/35942/

Camargo L.K.P.; Resende, J.T.V.; Mógor, A.F.; Camargo, C.K. ; Kurchaidt, S.M. (2016). Uso de índice de seleção na identificação de genótipos de batata doce com diferentes aptidões. Horticultura $\begin{array}{llll}\text { Brasileira } & 34 & (4): & 514-519 .\end{array}$ https://doi.org/10.1590/s0102-053620160410

Castro, L.; Becker, A. (2011). Normas e padrões para produção de mudas de batata-doce com alta sanidade. $1^{\circ}$ ed. Pelotas: Embrapa Clima Temperado. $37 \mathrm{p}$.

EMBRAPA. (2008). Batata doce (Ipomoea batatas). Embrapa Hortaliças: sistemas de produção. Disponível em: https://sistemasdeproducao.cnptia.embrapa.br/Fontes HTML/Batata-doce/Batatadoce_Ipomoea_batatas/apresentacao.html

Ferreira, D.F. (2011). Sisvar: a computer statistical analysis system. Ciência e Agrotecnologia (UFLA) 35 (6): 1039-1042. http://dx.doi.org/10.1590/S1413$\underline{70542011000600001}$

Ferreira, O.G.L.; Rossi, F.D.; Vaz, R.Z.; Fluck, A.C.; Costa, O.A.D.; Farias, P.P . (2017). Leaf area determination by digital image analysis. Archivos de 
$\begin{array}{llll}\text { Zootecnia } & 66 & \text { (256): } & 593-597 .\end{array}$ https://doi.org/10.21071/az.v66i256.2777

Filgueira, F. R. A. (2008). Novo manual de olericultura: agrotecnologia moderna na produção e comercialização de hortaliças. $3^{\circ} \mathrm{ed}$. Viçosa: Universidade Federal de Viçosa. 421p.

IBGE. (2019). Produção agrícola nacional de 2018. Instituto Brasileiro de Geografia e Estatística. Disponível

https://cidades.ibge.gov.br/brasil/pesquisa/14/0?indi $\underline{\text { cador }=10197 \& \text { localidade } 1=43}$

Lisboa, A.C.; Santos, P.S.; Neto, S.N.O.; Castro, D.N.; Abreu, A.H.M. (2012). Efeito do volume de tubetes na produção de mudas de Calophyllum brasiliense e Toona ciliata. Revista Árvore 36 (4): 603-609. https://doi.org/10.1590/S0100$\underline{67622012000400003}$

Melo, A. S. DE; Costa, B. C.; Brito, M. E. B.; Aguiar Netto, A. O.; Viégas, P. R. A. (2009). Custo e rentabilidade na produção de batata-doce nos perímetros irrigados de Itabaiana, Sergipe. Pesquisa Agropecuária Tropical (Agricultural Research in $\begin{array}{lllll}\text { the } & \text { Tropics) } & 39 & \text { (2): } & 119-123 .\end{array}$ https://doi.org/10.5216/pat.v39i2.3825

Mirasse, J. J. (2010). O consumo de batata-doce de polpa alaranjada entre famílias rurais do nordeste de Moçambique: um estudo sobre percepções de comida e Segurança Alimentar na província de Nampula. (Dissertação de mestrado). Universidade Federal do Rio Grande do Sul. Porto Alegre, RS, Brasil p. 13.

Neunfeld, T. H. (2019). Caracteres agronômicos, de pós-colheita e análise sensorial de acessos de batata-doce. (Tese de doutorado). Universidade Estadual do Centro-Oeste. Guarapuava, PR, Brasil p. 37.

Neumann, E.R.; Resende, J.T.V.; Camargo, L.K.P.; Chagas, R.R.; Lima Filho, R.B. (2017). Produção de mudas de batata doce em ambiente protegido com aplicação de extrato de Ascophyllum nodosum.

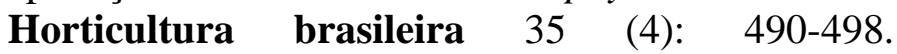
https://doi.org/10.1590/s0102-053620170404

Santana, A. D. D.; Arrigoni-Blank, M. F.; Blank, A. F.; Andrade, T. M.; Tavares, F. F.; Alves, R. P.; De
Santana A. D. (2015). Produção de mudas de batatadoce em função da posição da folha e de diferentes concentrações de AIB. Scientia Plena 11 (7): 1-10.

Sedam (2010). 21 Anos de Zoneamento Socioeconômico e Ecológico do Estado de Rondônia: Planejamento para o Desenvolvimento Sustentável e Proteção Ambiental. Disponível em: http://www.kaninde.org.br/wp-

content/uploads/2015/11/cartilha_zoneamento_inteir a_1332829095_1334545513.pdf

Silva, J. B. C. da; Lopez, C. A.; Miranda, J. E. C. de; Franca, F. H.; Carrijo, O. A.; Souza, A. F.; Pereira, W. (1995). Cultivo da batata-doce (Ipomoea batatas (L.) Lam). 3. ed. Brasília: EMBRAPA-CNPH. 18 p.

Reghin, M.Y.; Otto, R.F.; Olinik, J.R.; Jacoby, C.F.S. (2007). Viabilidade do sistema de produção de mudas em bandejas em três cultivares de cebola. Ciência e $\begin{array}{llll}\text { Agrotecnologia } & 31 & \text { (4): } & 1075-1084 .\end{array}$ http://dx.doi.org/10.1590/S1413-

$\underline{70542007000400020}$

Rós, A. B.; Araújo, H.S.; Narita, N. (2013). Uso de fertilizante de liberação lenta na produção de mudas de batata-doce em bandeja. Semina: Ciências $\begin{array}{llll}\text { Agrárias } & 34 & \text { (6): } & \text { 2667-2674. }\end{array}$ http://dx.doi.org/10.5433/1679$\underline{0359.2013 v 34 n 6 \mathrm{p} 2667}$

Rós, A. B.; Narita, N. (2011). Produção de mudas de batata-doce a partir de poucas plantas matrizes. Brazilian Journal of Agricultural Sciences 6 (1): 85-89. https://doi.org/10.5039/agraria.v6ila965

Rós-Golla A.; Silva, A.C. D.; Santos, V.B.; Araújo, H.S.; Fabri, E.G. (2008). Desenvolvimento inicial de raízes tuberosas de batata-doce (Ipomoea batatas (L.) em Presidente Prudente/SP. Horticultura Brasileira 26 (2): 244-247.

Vendruscolo, E.P.; Martins, A.P.B.; Campos, L.F.C.; Brandão, D.C.; Nascimento, L.M.; Seleguini, A. (2017). Produção de mudas de batata-doce de baixo custo em diferentes substratos e níveis de enfolhamento de estacas. Revista de Agricultura $\begin{array}{llll}\text { Neotropical } & 4 & \text { (2): } & \text { 102-109. }\end{array}$ https://doi.org/10.32404/rean.v4i2.1429 